\title{
C-VISA BikDD:Liposome
}

National Cancer Institute

\section{Source}

National Cancer Institute. C-VISA BikDD:Liposome. NCI Thesaurus. Code C88264.

A formulation composed of DOTAP:cholesterol liposome nanoparticles complexed with the plasmid C-VISA BiKDD, with potential antineoplastic activity. C-VISA BikDD: liposome consists of a pancreatic-cancer-specific expression vector VISA (VP16-GAL4-WPRE integrated systemic amplifier) and a pancreatic-cancer-specific promoter CCKAR (cholecystokinin type A receptor) (CCKAR-VISA or C-VISA) which drives expression of the gene BikDD, a mutant form of the potent proapoptotic gene Bik ( $\mathrm{BCl}-2$ interacting killer). Upon administration and transduction into pancreatic tumor cells, expression of BikDD by C-VISA BikDD:liposome may induce pancreatic tumor cell apoptosis and suppress pancreatic tumor cell proliferation. BikDD binds with greater affinity to anti-apoptotic proteins bcl-2, bcl-xl, bcl-w and $\mathrm{Mcl}-1$ and is more potent than wild-type Bik.

DOTAP:cholesterol liposome is composed of cationic lipid dioleoyl-trimethylammonium propane (DOTAP) and cholesterol at molar ratio of 1:1. 